\title{
Ensino Híbrido no Processo de Aprendizagem de Programação: Uma Revisão Sistemática
}

\author{
Josualdo Dias - IFBA Campus Porto Seguro - josualdodias@ifba.edu.br - \\ 0000-0002-1623-1546 \\ Rodolfo Seno Diaz - IFBA Campus Porto Seguro - rodosd@gmail.com - \\ 0000-0002-9677-9201
}

\begin{abstract}
Resumo. O Ensino Híbrido é uma abordagem pedagógica que combina atividades presenciais e atividades online, aprimorando a experiência de aprendizagem e integrando as tecnologias digitais à Educação. Com o objetivo de analisar seu uso em componentes curriculares de introdução à programação e identificar suas principais contribuições na aprendizagem dos alunos, foi realizada uma Revisão Sistemática da Literatura. O resultado da análise dos estudos levantados mostrou que o uso do Ensino Híbrido aumentou a motivação e a autonomia dos estudantes, promoveu a participação ativa e favoreceu a aprendizagem. Do mesmo modo, permitiu identificar diretrizes dos métodos, ferramentas e abordagens para planejar a implementação de um modelo híbrido eficaz.
\end{abstract}

Palavras-chave: ensino híbrido, tecnologias educacionais, ensino de programação

\section{Blended Learning in the Programming Learning Process: A Systematic Review}

Abstract. Blended Learning is a pedagogical approach that combines face-to-face activities and online activities, enhancing the learning experience and integrating digital technologies into Education. In order to analyze its use in curricular components for introductory programming and identify its main contributions to student learning, a Systematic Literature Review was carried out. The result of the analysis of the surveyed studies showed that the use of Blended Learning increased the motivation and autonomy of students, promoted active participation and favored learning. Likewise, it allowed the identification of guidelines for methods, tools and approaches to plan the implementation of an effective hybrid model.

Keywords: blended learning, educational technologies, programming teaching

\section{Introdução}

O ensino de programação tem como objetivo o desenvolvimento das capacidades do aluno, adquirindo competências e conhecimentos necessários para conceber sistemas que permitam resolver problemas da vida real [Garlet et al. 2016]. Esta competência vem sendo considerada como um dos pilares fundamentais do intelecto humano, junto com a leitura, a escrita e a aritmética pois serve para descrever, explicar e modelar o universo e seus processos complexos [SBC 2019].

No entanto, a aprendizagem de programação exige alto grau de abstração, além de tempo e esforço de aprendizagem, representando um desafio constante para professores e alunos. De acordo com [Gomes 2010], a experiência tem demonstrado que existe uma grande dificuldade em compreender a aplicação de certos conceitos da programação 
por parte de uma percentagem significativa de alunos que frequentam disciplinas introdutórias nesta área. A preocupação pelos altos índices de insucesso nesses componentes curriculares tem motivado debates e pesquisas das quais surgiram muitas propostas que, no entanto, não provocaram as melhorias previstas.

Nesse contexto, o ensino híbrido propõe uma experiência de aprendizagem na qual convergem o modelo de ensino presencial, que vem sendo utilizado tradicionalmente, e o modelo on-line, que utiliza as tecnologias digitais para promover a aprendizagem, buscando usufruir as vantagens de cada modalidade [Horn e Staker 2015]. Esses dois ambientes de aprendizagem tornam-se complementares, já que além do uso de variados recursos digitais, o indivíduo interage com os pares e os professores, intensificando a troca de experiências que acontecem na sala de aula.

Diante do exposto, esta pesquisa teve como principal objetivo analisar o uso do ensino híbrido em disciplinas de introdução à programação no ensino superior. Para tal fim, foi realizada uma Revisão Sistemática da Literatura que analisou estudos publicados sobre essa temática em diferentes regiões, durante os últimos anos.

Essa primeira seção apresentou a introdução do trabalho junto com seus objetivos. A segunda seção descreve a metodologia e os procedimentos utilizados para a Revisão Sistemática. Na terceira seção, são apresentados os resultados e discussões. Finalmente, na quarta seção são apresentadas as considerações finais.

\section{Metodologia}

Esta pesquisa foi desenvolvida seguindo as diretrizes para elaboração de revisões sistemáticas propostas por [Kitchenham 2004], que adaptou guias utilizadas por pesquisadores médicos para atender problemas da pesquisa da área da Engenharia de Software. Segundo a autora, uma revisão sistemática da literatura é um meio de identificar, avaliar e interpretar todas as pesquisas disponíveis relevantes para uma questão de pesquisa específica, área de tópico ou fenômeno de interesse. Esse procedimento resume os estágios de uma revisão sistemática em três fases principais: Planejamento, Condução e Apresentação de Resultados.

De acordo com [Sampaio e Mancini 2006], uma boa revisão sistemática requer uma pergunta bem formulada e clara. Nesse sentido, este trabalho busca responder às questões de pesquisa (QP) a seguir:

QP1. Quais são as principais ferramentas e softwares utilizados na aplicação dos modelos de ensino híbrido?

QP2. Quais foram as metodologias de ensino utilizadas e como se relacionam com a busca pela personalização do ensino e a motivação dos alunos?

QP3. Quais são os processos avaliativos validados no uso dos modelos de ensino híbrido? QP4. Quais são os benefícios e limitações da aplicação de modelos de ensino híbrido para a aprendizagem de programação?

\subsection{Planejamento}

A primeira etapa da revisão sistemática consistiu no planejamento do estudo, delimitando o tema, o escopo e os objetivos, de forma de orientar cada uma das fases seguintes. 
O termo de busca foi derivado das questões de pesquisa através de variações das palavras-chave. Após testar algumas variantes se optou por utilizar uma estratégia de busca com os termos principais, com o propósito de obter uma maior quantidade de estudos retornados. Desta forma, a string ficou definida da seguinte forma:

("Ensino Híbrido"OR "Educação Híbrida"OR "Aprendizagem Mista"OR "Ensino Personalizado") AND ("Introdução à Programação"OR "Curso de Programação")

Com o intuito de realizar uma busca exaustiva, foram definidas quatro bases de dados (ACM Digital Library, DOAJ, ERIC, IEEE Xplore Digital Library) onde se aplicou a busca automatizada, utilizando os recursos das próprias bases junto com o termo de busca. Além delas foi realizada a busca em duas conferências (Simpósio Brasileiro de Informática na Educação, Workshop de Informática na Escola) onde se realizou de forma manual, com uma leitura prévia dos resumos e palavras-chave. Essas fontes foram escolhidas por indexarem alguns veículos qualificados da área de Ciência da Computação e Educação.

Ainda nessa fase foram definidos os critérios de inclusão (CI) e exclusão (CE) necessários para dar início à revisão sistemática:

CI1. Produções publicadas no período de 2016 até 2020.

CI2. Estudos escritos em português, inglês ou espanhol.

CI3. Trabalhos que abordam a relação de modelos de ensino híbrido com a aprendizagem de programação.

CI4. Trabalhos relacionados à personalização do ensino.

CI5. Produções que abordam a educação no ensino superior.

CE1. Estudos secundários: relatórios técnicos, revisão da literatura, shortpaper, opinion paper, resumos estendidos.

CE2. Publicações que não condizem em responder às questões da pesquisa.

CE3. Trabalhos de acesso restrito ou não disponíveis para download em redes domésticas.

CE4. Estudos repetidos em bases de dados diferentes.

CE5. Publicações com menos de 5 páginas ou mais de 12 páginas de acordo com os padrões da ABNT.

\subsection{Condução}

Neste estágio da pesquisa foram realizadas as buscas, seleção e classificação dos dados principais dos estudos levantados. Esta etapa foi executada no período entre janeiro e março de 2021.

Em conformidade com os parâmetros definidos no protocolo da revisão sistemática, foram selecionados 25 estudos primários, conforme mostra a tabela 1 .

Após a análise dos estudos obtidos nas buscas, foi realizada a extração dos dados dos trabalhos selecionados. Desse modo, para armazenar as informações e facilitar a interpretação, foram organizados os principais dados de cada estudo em uma planilha eletrônica. 
Tabela 1. Resultados da busca e aplicação dos critérios de seleção.

\begin{tabular}{|c|c|c|c|}
\hline Base de Dados & Total de trabalhos & Pré-selecionados & Selecionados \\
\hline ACM & 121 & 15 & 7 \\
\hline DOAJ & 207 & 7 & 4 \\
\hline ERIC & 37 & 8 & 4 \\
\hline IEEE & 28 & 6 & 5 \\
\hline SBIE & 6 & 6 & 2 \\
\hline WIE & 4 & 4 & 3 \\
\hline Totais & $\mathbf{4 0 3}$ & $\mathbf{4 6}$ & $\mathbf{2 5}$ \\
\hline
\end{tabular}

\section{Resultados}

A partir da síntese dos dados buscou-se organizar as informações levantadas para responder às questões de pesquisa definidas no escopo do trabalho. Com o propósito de referenciar os estudos primários selecionados no decorrer da análise dos dados, cada publicação foi identificada com um código, conforme apresentado na tabela 2.

\subsection{Ferramentas e Softwares}

Em primeiro lugar, atendendo à QP1, foi evidenciada a relevância do uso de Ambientes Virtuais de Aprendizagem (AVA) na abordagem de ensino estudada. Desta forma, os AVA e suas funcionalidades foram mencionados na maior parte dos estudos selecionados. A preferência pelo Moodle foi justificada por ser uma plataforma de código aberto que pode ser hospedada em um servidor dedicado dentro da própria instituição, o que dá maior controle sobre os dados armazenados. Assim mesmo, permite que os educadores criem os conteúdos e currículos de forma completa usando uma variedade de plugins gratuitos, como o Virtual Programming Lab (VPL), usado para executar atividades e submeter programas obtendo avaliação automática sem a necessidade de instalar e configurar ferramentas de programação [Demaidi et al. 2019]. Por outro lado, foi citado nos estudos o uso dos juízes online no ensino de programação. Esses sistemas permitem a correção automática de códigos desenvolvidos pelos usuários como resposta a determinados exercícios de programação, comparando a saída retornada com a saída esperada [Galvão et al. 2016]. Além de ser uns dos recursos oferecidos pelo já mencionado VPL [E9], o uso de outros juízes online são relatados nos artigos analisados, tais como URI, UVA, Code Chef, HackerRank, run.codes, BOCA Online Contest Administrator [E21], [E22], QBASIC Programming Achievement Test (QBPAT) [E1], SmartApe [E2], TryHaskell [E10] e TRAKLA2 [E13].

Do mesmo modo, a relevância da produção de vídeo aulas para o ensino online foi destacada em 15 dos trabalhos analisados [E2], [E4], [E5], [E7], [E8], [E9], [E10], [E11], [E12], [E14], [E15], [E17], [E18], [E20], [E24]. Através dessas demonstrações de vídeo os professores podem expor os conteúdos da disciplina e incluir simulações de programação para que alunos acompanhem [Sharp e Sharp 2017]. [Zeuch 2019] destacam a importância da interatividade nos vídeos e indicam que a duração não deve exceder os 15 minutos, sugerindo a inclusão de questionários ou exercícios para criar interrupções e manter a atenção dos alunos. 
Tabela 2. Estudos selecionados.

\begin{tabular}{|c|c|c|}
\hline Id & Título & Autor e Ano \\
\hline E1 & $\begin{array}{l}\text { Effects of B-learning and F2F learning environments on students } \\
\text { achievement in QBASIC programming }\end{array}$ & [Olelewe e Agomuo 2016] \\
\hline $\mathbf{E 2}$ & $\begin{array}{l}\text { Experiences in introducing blended learning in an introductory } \\
\text { programming course }\end{array}$ & [Albrecht et al. 2018] \\
\hline $\mathbf{E 3}$ & First step in implementing pedagogy of integration using Edmodo & [Ryane e El Faddouli 2018] \\
\hline $\mathbf{E 4}$ & $\begin{array}{l}\text { Game changing mobile learning based method mix for teaching } \\
\text { software development }\end{array}$ & [Schefer e Miladinovic 2017] \\
\hline $\mathbf{E 5}$ & $\begin{array}{l}\text { Longitudinal data on flipped class effects on performance in CS1 } \\
\text { and retention after CS1 }\end{array}$ & [Latulipe et al. 2018] \\
\hline E6 & $\begin{array}{l}\text { Personalized contents based on cognitive level of student's com- } \\
\text { putational thinking for learning basic competencies of program- } \\
\text { ming using an environment blearning }\end{array}$ & [López e García-Peñalvo 2016] \\
\hline E7 & The case of the fragmented classroom & [Billingsley 2019] \\
\hline $\mathbf{E 8}$ & $\begin{array}{l}\text { A case study of using edmodo to enhance computer science lear- } \\
\text { ning for engineering students }\end{array}$ & [Ryane e El Faddouli 2020] \\
\hline E9 & $\begin{array}{l}\text { Applying educational data mining to explore students' learning } \\
\text { patterns in the flipped learning approach for coding education. }\end{array}$ & [Hung et al. 2020] \\
\hline E10 & $\begin{array}{l}\text { Learner experiences of a blended course incorporating a MOOC } \\
\text { on Haskell functional programming }\end{array}$ & [Dale e Singer 2019] \\
\hline E11 & Students in Blended Learning By Flipped Classroom Approach & [Nedeva et al. 2019] \\
\hline E12 & $\begin{array}{l}\text { A Comparision of Student Academic Performance with Tradicio- } \\
\text { nal, Online and Flipped Instructional Approaches in C\# Program- } \\
\text { ming Course }\end{array}$ & [Sharp e Sharp 2017] \\
\hline E13 & $\begin{array}{l}\text { An In-Depth Analysis of Teaching Themes and the Quality of } \\
\text { Teaching in Higher Education: Evidence from the Programming } \\
\text { Education Environments. }\end{array}$ & [Xia 2017] \\
\hline E14 & $\begin{array}{l}\text { Flipping a Programing Class to Improve Student Performance } \\
\text { and Student Satisfaction }\end{array}$ & [Chen e Faichney 2019] \\
\hline E15 & $\begin{array}{l}\text { Teaching Strategies and Methods in Modern Environments for } \\
\text { Learning of Programming }\end{array}$ & [Djenic e Mitic 2017] \\
\hline E16 & Applying blended learning in programming courses & [Demaidi et al. 2019] \\
\hline E17 & $\begin{array}{l}\text { Evaluation of a re-designed introductory course "Programming in } \\
\text { C" with video support }\end{array}$ & [Zeuch 2019] \\
\hline E18 & $\begin{array}{l}\text { Introducing Basic Programming to Pre-University Students : A } \\
\text { Successful Initiative in Singapore }\end{array}$ & [Mok e Rao 2018] \\
\hline E19 & $\begin{array}{l}\text { Study of a pedagogy adopted to generate interest in students ta- } \\
\text { king a programming course }\end{array}$ & [Venugopal-Wairagade 2016] \\
\hline E20 & Flipped classroom in a CS1 course & [Kereki e Adorjan 2020] \\
\hline $\mathbf{E 2 1}$ & $\begin{array}{l}\text { Análise de modelo para a tomada de decisões pedagógicas em um } \\
\text { ambiente voltado ao aprendizado de algoritmos }\end{array}$ & [Maranhão et al. 2020] \\
\hline $\mathbf{E 2 2}$ & $\begin{array}{l}\text { Juiz online como ferramenta de apoio a uma metodologia de en- } \\
\text { sino híbrido em programação }\end{array}$ & [Galvão et al. 2016] \\
\hline $\mathbf{E 2 3}$ & $\begin{array}{l}\text { Ensino de Introdução à Programação na Modalidade Semipresen- } \\
\text { cial com o Apoio do Google Classroom }\end{array}$ & [Santos et al. 2019] \\
\hline $\mathbf{E 2 4}$ & $\begin{array}{l}\text { ML-SAI: Um Modelo Pedagógico para Atividades de M- } \\
\text { Learning que Integra a Abordagem da Sala de Aula Invertida }\end{array}$ & [Martins e Gouveia 2019] \\
\hline $\mathbf{E 2 5}$ & $\begin{array}{l}\text { Pensamento Computacional Praticado com um Jogo Casual Sério } \\
\text { no Ensino Superior }\end{array}$ & [Vahldick et al. 2016] \\
\hline
\end{tabular}




\subsection{Metodologias e Personalização do Ensino}

Em reposta à QP2, buscou-se analisar a contribuição das metodologias de ensino utilizadas para promover a personalização do ensino e a motivação dos alunos na aprendizagem de programação.

A forma de classificação das abordagens de ensino foi baseada nas categorizações feitas pelos autores dos estudos. É importante salientar que as categorias mencionadas não são mutuamente exclusivas, podendo ser abordadas combinando modelos. A figura 1 apresenta a lista dos métodos de ensino citados nos estudos e as combinações utilizadas.

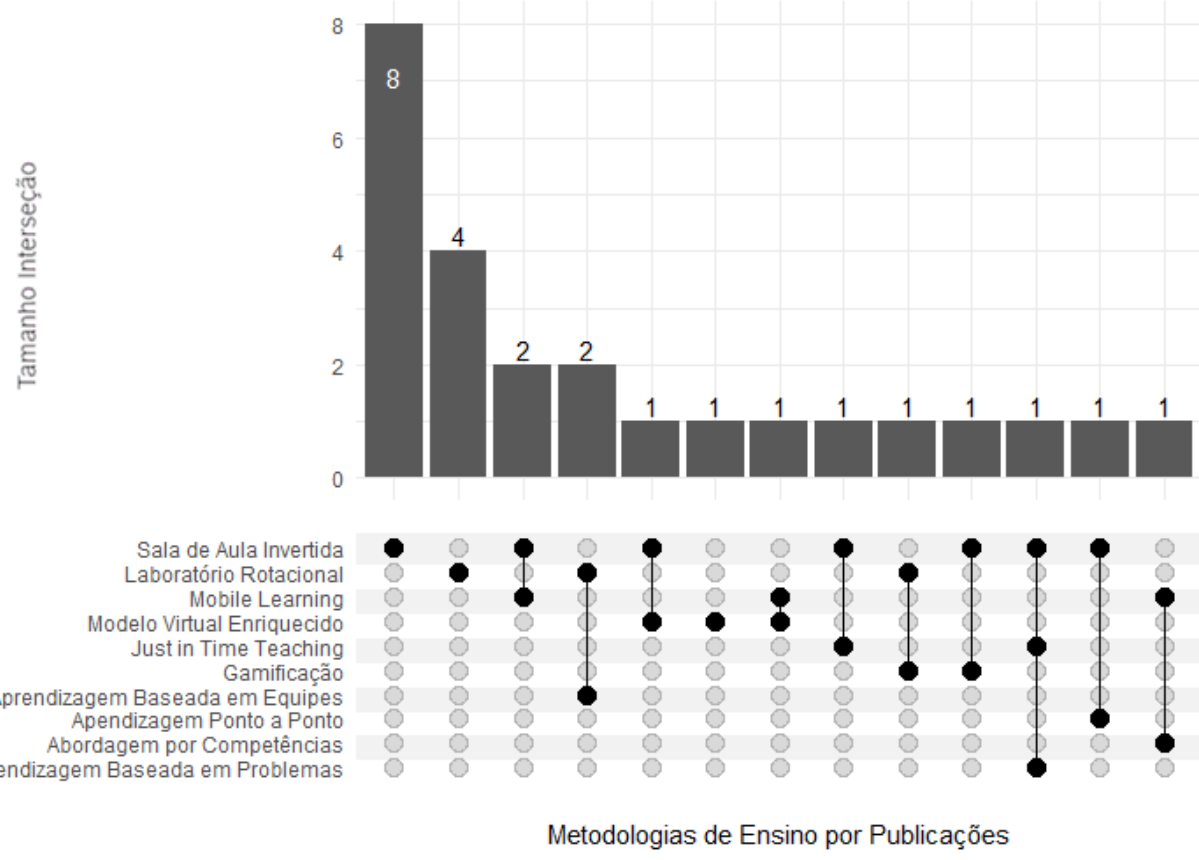

Figura 1. Métodos de Ensino associados ao Ensino Híbrido.

Dentre os métodos citados, o de sala de aula invertida foi o mais utilizado [E1], [E4], [E5], [E6], [E9], [E10], [E11], [E12], [E14], [E17], [E18], [E19], [E20], [E23], [E24]. Nesse sentido, [Nedeva et al. 2019] afirmam que nesse modelo o tempo normalmente gasto com palestras é usado para atividades em sala de aula, discussões, problemas e projetos em grupo. Como resultado do uso eficiente do tempo extra da aula, a aprendizagem torna-se mais significativa. Por outro lado, [Djenic e Mitic 2017] complementam que o planejamento pedagógico moderno deve incorporar multimídia interativa e interação no ensino, combinando aprendizagem colaborativa e autodirigida, de forma que o aluno desenvolva a criatividade e adquira habilidades para resolução de problemas e desenvolvimento de tecnologias.

Em relação à personalização do ensino de programação em modelos híbridos, 16 estudos abordam a questão [E1], [E2], [E4], [E6], [E7], [E8], [E9], [E10], [E13], [E14], [E15], [E16], [E18], [E22], [E24], [E25]. Esse processo acontece quando a aprendizagem é adaptada às necessidades particulares dos estudantes. De acordo com [Billingsley 2019], o design dos cursos deve considerar as diferentes formas de variação dentro da classe, para que além de saber lidar com elas, possam ser transformadas em 
uma vantagem da diversidade. Do mesmo modo, [Maranhão et al. 2020] salientam a relevância do aporte dos sistemas inteligentes para esse fim. Mesmo que essas ferramentas não consigam determinar o estado de conhecimento do aluno com exatidão, são capazes de fazer uma série de suposições com base nas respostas submetidas pelos alunos, fornecendo informações relevantes para os professores e proporcionando recomendações para os alunos.

\subsection{Processos Avaliativos}

Com o propósito de responder a QP3, forma verificados e analisados os processos avaliativos utilizados nas abordagens de ensino híbrido.

Entre os mencionados, destacam-se as atividades online e os exames presenciais, os quais aparecem frequentemente combinados. Nesse sentido, um modelo híbrido pode propor que após as aulas presenciais, incluam-se atividades de revisão online junto com avaliações formativas, de modo a garantir que a revisão seja bemsucedida, ou em caso contrário, encaminhar o aluno para uma atividade de recuperação [Ryane e El Faddouli 2018]. Assim mesmo, para uma aprendizagem eficaz, é importante que os alunos possam acompanhar seu próprio progresso, e os recursos de avaliação automática fornecem uma rápida estimativa do estado de conhecimento de um estudante [Albrecht et al. 2018]. Por outro lado, o uso de atividades colaborativas em laboratório como recurso avaliativo também evidenciou sua relevância no estímulo de discussões entre os alunos. Junto com a avaliação entre pares, o principal objetivo destas avaliações qualitativas é o de proporcionar aos alunos a oportunidade de refletir sobre o seu progresso nas competências de programação e permitir uma experiência de aprendizagem mais motivadora, levando consequentemente, a melhores resultados [Schefer e Miladinovic 2017].

Mesmo com os dados expostos, considerou-se que os estudos explorados não descreveram os processos avaliativos de forma detalhada. Desse modo, a análise de resultados tornou-se mais complexa e prejudicou a possibilidade de reprodução dos métodos utilizados em trabalhos futuros.

\subsection{Contribuições e Limitações}

Como resultado desta pesquisa, atendendo à $\mathbf{Q P 4}$ foi possível evidenciar as contribuições e limitações do ensino híbrido para o processo de ensino aprendizagem.

Em primeiro lugar, ao colocar o aluno no centro do processo de aprendizagem, os modelos híbridos buscam se aproximar da perspectiva dos estudantes e assim obter um planejamento eficaz que gere maior disposição para aprender. Dessa forma, a motivação, o entusiasmo e o estímulo dos alunos no processo de aprendizagem são amplamente discutidos em todas as publicações selecionadas desta revisão sistemática. [Ryane e El Faddouli 2020] afirmam que o Ensino Híbrido facilita a experiência de aprendizagem independente e colaborativa, oferece melhores resultados de aprendizagem, possibilita a criação de comunidades de investigação e aumenta a satisfação dos alunos. Além disso, o uso de metodologias ativas incentiva que os alunos aprendam de forma autônoma e participativa. Do mesmo modo, [Dale e Singer 2019] destacam que o principal benefício para os alunos foi a flexibilidade oferecida pelo formato híbrido. Por outro lado, foi evidenciada a relevância da obtenção de dados educacionais a partir das atividades online para análise e construção do conhecimento. Este tipo de informação é crucial 
para estudar o comportamento do aluno e prever o seu desempenho [Hung et al. 2020]. Além disso, com uso dos software educacionais que provêm feedback automático para corrigir programas submetidos, são estimulados o aprendizado e a autonomia dos alunos.

Em relação às limitações e desafios identificados nas experiências do uso dos métodos híbridos, 5 estudos mencionaram que as constantes revisões das metodologias, atualizações dos conteúdos e preparo do ambiente, demandam uma quantidade de tempo maior se comparado ao tradicional [E13], [E15], [E17], [E18], [E20]. Desse modo, [Kereki e Adorjan 2020] afirmam que os cursos a serem adaptados ao ensino híbrido precisam de uma revisão completa e do apoio das autoridades e de especialistas em design instrucional. Além do mais, os educadores devem ser preparados de forma abrangente já que o ensino em ambientes híbridos requer abordagens diferentes do ensino presencial e exige habilidades para a integração de ferramentas digitais.

\section{Considerações Finais}

A partir desta Revisão Sistemática da Literatura evidenciou-se que a abordagem de ensino híbrido apresenta um aprimoramento nos seus resultados quando está associada a metodologias ativas como a sala de aula invertida. Já os métodos avaliativos, mantiveram o exame presencial combinado com atividades online individuais e colaborativas, como os mais utilizados.

De acordo com o observado nesta pesquisa, a quantidade de estudos sobre o uso do ensino híbrido nos componentes curriculares de programação ainda é relativamente baixa, principalmente a nível nacional e regional. Além disso, foi identificada uma lacuna em relação ao estudo dos processos avaliativos, considerando que as publicações não descreveram os métodos utilizados de forma precisa.

Entretanto, os trabalhos analisados revelaram uma visão geral que permitiu identificar padrões em relação à aplicação da abordagem híbrida. Nesse sentido, e conforme apresentado nos resultados, foi possível reconhecer diretrizes dos métodos, ferramentas e abordagens para planejar a implementação de um modelo híbrido eficaz.

Mesmo sendo de diversas regiões do mundo, os estudos apresentaram como principal justificativa, as dificuldades de aprendizagem que o ensino de programação ocasiona em grande parte dos alunos. Para diminuir esses efeitos é proposto um modelo de ensino centrado no aluno, combinando atividades online e presenciais, individuais e colaborativas e criando um ambiente estruturado com aprendizagem ativa.

\section{Referências}

Albrecht, E., Gumz, F., e Grabowski, J. (2018). Experiences in introducing blended learning in an introductory programming course. ACM International Conference Proceeding Series, pages 93-101.

Billingsley, W. (2019). The case of the fragmented classroom. Proceedings - 2019 IEEE/ACM 41st International Conference on Software Engineering: Software Engineering Education and Training, pages 74-83.

Chen, D. e Faichney, J. (2019). Flipping a Programing Class to Improve Student Performance and Student Satisfaction. International Journal of Adult Vocational Education and Technology, 10(1):27-39. 
Dale, V. H. e Singer, J. (2019). Learner experiences of a blended course incorporating a MOOC on Haskell functional programming. Research in Learning Technology, 27:115 .

Demaidi, M. N., Qamhieh, M., e Afeefi, A. (2019). Applying blended learning in programming courses. IEEE Access, 7:156824-156833.

Djenic, S. e Mitic, J. (2017). Teaching strategies and methods in modern environments for learning of programming. 14th International Conference on Cognition and Exploratory Learning in the Digital Age, pages 189-196.

Galvão, L., Fernandes, D., e Gadelha, B. (2016). Juiz online como ferramenta de apoio a uma metodologia de ensino híbrido em programação. Anais do XXVII Simpósio Brasileiro de Informática na Educação, 27(1):140.

Garlet, D., Bigolin, N. M., e Silveira, S. R. (2016). Uma proposta para o ensino de programação de computadores na educação básica.

Gomes, A. d. J. (2010). Dificuldades de aprendizagem de programação de computadores: contributos para sua compreensão e resolução. Doutorado em engenharia informática, Universidade de Coimbra, Coimbra, Portugal.

Horn, M. e Staker, H. (2015). Blended: usando a inovação disruptiva para aprimorar a educação. Penso, Porto Alegre.

Hung, H. C., Liu, I. F., Liang, C. T., e Su, Y. S. (2020). Applying educational data mining to explore students' learning patterns in the flipped learning approach for coding education. Symmetry, 12(2):1-14.

Kereki, I. F. D. e Adorjan, A. (2020). Flipped classroom in a CS1 course. IEEE Global Engineering Education Conference, EDUCON, 2020-April:110-114.

Kitchenham, B. (2004). Procedures for performing systematic reviews. Joint Technical Report, 37(1):79-83.

Latulipe, C., Rorrer, A., e Long, B. (2018). Longitudinal data on flipped class effects on performance in CS1 and retention after CS1. SIGCSE 2018 - Proceedings of the 49th ACM Technical Symposium on Computer Science Education, 2018-Janua:411-416.

López, A. R. e García-Peñalvo, F. J. (2016). Personalized contents based on cognitive level of student's computational thinking for learning basic competencies of programming using an environment blearning. ACM International Conference Proceeding Series, 02-04-Nove:1139-1145.

Maranhão, D., Raposo, A., e Neto, C. S. (2020). Análise de modelo para a tomada de decisões pedagógicas em um ambiente voltado ao aprendizado de algoritmos. In Anais do XXXI Simpósio Brasileiro de Informática na Educação, pages 1223-1232, Porto Alegre, RS, Brasil. SBC.

Martins, E. e Gouveia, L. (2019). ML-SAI: um modelo pedagógico para atividades de m-learning que integra a abordagem da sala de aula invertida. In Anais do XXVII Workshop sobre Educação em Computação, pages 121-130, Porto Alegre, RS, Brasil. SBC. 
Mok, H. N. e Rao, V. R. (2018). Introducing basic programming to pre-university students: A successful initiative in Singapore. 17th International Conference on Information Technology Based Higher Education and Training (ITHET), pages 1-7.

Nedeva, V., Dineva, S., e Ducheva, Z. (2019). Students in blended learning by flipped classroom approach. Information Technologies and Learning Tools, 72(4):204-213.

Olelewe, C. J. e Agomuo, E. E. (2016). Effects of b-learning and f2f learning environments on students' achievement in qbasic programming. Computers \& Education, 103:76-86.

Ryane, I. e El Faddouli, N. E. (2018). First step in implementing pedagogy of integration using Edmodo. ACM International Conference Proceeding Series.

Ryane, I. e El Faddouli, N. E. (2020). A case study of using edmodo to enhance computer science learning for engineering students. International Journal of Emerging Technologies in Learning, 15(3):62-73.

Sampaio, R. e Mancini, M. (2006). Estudos de revisão sistemática: Um guia para síntese criteriosa da evidência científica. Revista Brasileira de Fisioterapia, pages 83-89.

Santos, H., Souza, F., Santos, B., Amorim, I., Silva, M., e Filho, A. (2019). Ensino de introdução à programação na modalidade semipresencial com o apoio do google classroom. In Anais do XXVII Workshop sobre Educação em Computação, pages 131140, Porto Alegre, RS, Brasil. SBC.

SBC, S. B. d. C. (2019). Diretrizes para ensino de computação na educação básica.

Schefer, S. W. e Miladinovic, I. (2017). Game changing mobile learning based method mix for teaching software development. ACM International Conference Proceeding Series.

Sharp, J. H. e Sharp (2017). A comparison of student academic performance with traditional, online, and flipped instructional approaches in a c programming course. Journal of Information Technology Education, 16:142-155.

Vahldick, A., Mendes, A., Marcelino, M., e Farah, P. (2016). Pensamento computacional praticado com um jogo casual sério no ensino superior. In Anais do XXIV Workshop sobre Educação em Computação, pages 308-317, Porto Alegre, RS, Brasil. SBC.

Venugopal-Wairagade, G. (2016). Study of a pedagogy adopted to generate interest in students taking a programming course. International Conference on Learning and Teaching in Computing and Engineering, pages 141-146.

Xia, B. S. (2017). An In-Depth Analysis of Teaching Themes and the Quality of Teaching in Higher Education: Evidence from the Programming Education Environments. International Journal of Teaching and Learning in Higher Education, 29(2):245-254.

Zeuch, K. (2019). Evaluation of a re-designed introductory course " Programming in C " with video support. 2019 18th International Conference on Information Technology Based Higher Education and Training (ITHET), pages 1-6. 